\title{
Acuerdos de pesca en los lagos de Tarapoto: alternativa de gestión para los bienes comunes en la Amazonia colombiana
}

\section{Fisheries agreements in the Lakes of Tarapoto: alternative management for common goods in the Colombian Amazon.}

\author{
Acordos de pesca nos lagos de Tarapoto: alternativa de \\ Gestão para os bens comuns na Amazônia Colombiana
}

\author{
Catalina Trujillo Osorio', Liseth Johanna Escobar² \& Fernando Trujillo González ${ }^{3}$ \\ 'Economista, Magister en Estudios Amazónicos. 'Economista, Candidata a Magister en Estudios Amazónicos. \\ ${ }^{3}$ Biólogo Marino, Magister en Ciencias, Doctor en Zoología \\ ${ }^{1}$ Facultad de Ciencias Económicas, Universidad del Quindío. \\ ${ }^{2}$ Universidad Nacional de Colombia, Sede Amazonía. ${ }^{3}$ Fundación Omacha \\ ${ }^{1}$ ctrujillo@uniquindio.edu.co, ${ }^{2}$ ljescobara@unal.edu.co, ${ }^{3}$ fernando@omacha.org
}

\section{Resumen}

Se presentan las experiencias de gestión de recursos pesqueros en resguardos indígenas del Amazonas colombiano y los resultados y limitaciones del proceso adelantado para la construcción de acuerdos comunitarios de pesca. Se usó la metodología de investigación-acción-participación respondiendo al objetivo de crear estrategias para contrarrestar los problemas de agotamiento del recurso pesquero de los lagos de Tarapoto en el resguardo Ticoya y consolidar estrategias propias para fortalecer la capacidad de gobernanza interna del recurso pesquero dentro del territorio indígena. Se encontró que los acuerdos de pesca actúan como instituciones que regulan y optimizan el uso de bienes comunes de libre acceso, y consolidan la capacidad de gobernanza en territorios indígenas. Se concluye que la consolidación de un sistema propio de gestión debe ir más allá del control del recurso, evidenciando los esfuerzos locales por adoptar sistemas de manejo alternativo que se integren al modo de vida indígena y a los modelos de producción tradicional.
Palabras clave: cuenca amazónica, gestión pesquera, gobernanza pesquera, pesca artesanal, propiedad colectiva.

\section{Abstract}

The experiences of management of fish resources in indigenous safeguards of the Colombian Amazon and the results and limitations of the advance process for the construction of Community fishing agreements are presented. The methodology of research-actionparticipation was used responding to the objective of creating strategies to counteract the problems of depletion of the fishing resource of the lakes of Tarapoto in the Ticoya shelter and to consolidate strategies of its own to To strengthen the internal governance capacity of the fishing resource within the indigenous territory. It was found that fisheries agreements act as institutions that regulate and optimize the use of common free-access goods, and consolidate governance capacity in indigenous territories. It was 
concluded that the consolidation of a management system should go beyond the control of the resource, evidencing local efforts to adopt alternative management systems that integrate the indigenous way of life and traditional production models.

Key-words: Amazon basin, fisheries management, fisheries governance, artisanal fisheries, collective property.

\section{Resumo}

Apresentamos as experiências de gestão de recursos haliêuticos nas reservas indígenas da Amazônia colombiana e os resultados e limitações do processo avançado para a construção de acordos de pesca comunitários. A metodologia pesquisa-açãoparticipação foi utilizada em resposta ao objetivo de criar estratégias para combater os problemas de esgotamento dos recursos pesqueiros dos lagos de Tarapoto no abrigo de Ticoya e consolidar suas próprias estratégias para fortalecer a capacidade interna de governança do recurso pesqueiro dentro da território indígena. Verificou-se que os acordos de pesca atuam como instituições que regulam e otimizam o uso de bens comuns de livre acesso e consolidam a capacidade de governança em territórios indígenas. Conclui-se que a consolidação de um próprio sistema de gestão deve ultrapassar o controle do recurso, evidenciando os esforços locais para adotar sistemas de gerenciamento alternativos integrados ao estilo de vida indígena e modelos de produção tradicionais.

Palavras chave: bacia amazônica, gestão das pescas, governança das pescarias, pesca artesanal, propriedade coletiva.

\section{Introducción}

La cuenca amazónica contiene la mayor riqueza íctica entre los sistemas de agua dulce del planeta (Gutierrez, 2010), por lo que la pesca es la principal fuente de alimento de la sociedad amazónica (Pantevis Girón, 2013), el canal central de seguridad alimentaria de la población nativa (Trujillo \& Florez, 2016), y una de las mayores actividades económicas de la región (Prieto, 2000; Universidad Nacional de Colombia, Corpoamazonia, Fundación Omacha, 2007; Barreto \& Borda, 2008). Es por ello que la conservación y el control de la actividad pesquera se ha tornado escencial para la sostenibilidad socioambiental, evidenciando la importancia de consolidar sistemas altenativos de manejo para los recursos pesqueros amazónicos (Instituto de Investigación de los Recursos Hidrobiológicos Alexander von Humboldt, 2011).

Este artículo presenta las experiencias de la comunidad indígena del municipio de Puerto Nariño en el Amazonas Colombiano, para consolidar un sistema de gestión alternativo basado en la implementación de acuerdos de pesca propios, planteados a partir del conocimiento local de los pescadores sobre las dinámicas del ciclo hídrico amazónico y las necesidades de las especies ícticas de mayor uso pesquero (Trujillo \& Trujillo, 2009). En este contexto, los acuerdos de pesca como instrumentos de gestión ambiental se conciben como reglas locales creadas por los habitantes del territorio con el objetivo de dirigir y controlar las extracción de los recursos pesqueros dentro del mismo, ratificando el derecho de autogestión de los usuarios, sobre los bienes comunes de su terriotorio (Castello, 2013).

Con este mismo enfoque, en la Amazonia se han realizado otras experiencias de acuerdos de pesca, donde las comunidades ribereñas han implementado sistemas de manejo pesquero basado en reglas de juego locales. Estos procesos han sido bien desarrollados en la Amazonia Brasilera, donde por ejemplo en la región de Tefe, Estado do Amazonas, se definieron y delimitaron lagos de reserva, de preservación y lagos de libre uso; así mismo en la región de Santarém, Estado do Pará, los acuerdos y reglas de uso generadas por los pescadores 
locales organizados fueron respaldadas legalmente por la autoridad ambiental brasilera IBAMA, por lo que comenzaron a tener un cumplimiento de tipo obligatorio (ProVarzea, 2005).

En el caso de la Amazonia colombiana, algunas experinecias de manejo se encuentran en Guainía en el río Inírida-Estrella Fluvial del Inírida (Zuluaga \& Franco, 2014), en el bajo río Caquetá- La Pedrera (Palma, 2014), y en el departamento del Amazonas, en Leticia con los acuerdos de los lagos de Yahuarcaca (Universidad Nacional de Colombia Sede Amazonia, 2011), y en Puerto Nariño en los lagos de Tarapoto (Trujillo \& Trujillo, 2009). Entre este grupo de experiencias, los acuerdos de pesca de los lagos de Tarapoto se constituyen como el proceso de manejo local de recursos pesqueros con mayor antigüedad en el país.

Los lagos de Tarapoto se localizan en el resguardo indígena Ticuna-Cocama-Yagua (Ticoya), del Municipio de Puerto Nariño, departamento del Amazonas Colombiano (Moreno, 2014). El municipio tiene un area de $1.074 \mathrm{Km}^{2}, 92 \%$ perteneciente al resguardo, dentro de los cuales $8,9 \%$ constituye los humedales y lagos de Tarapoto (Trujillo \& Duque, 2014); acorde al pensamiento indigena estos representan el origen de la vida y la cultura del pueblo Ticoya (Camacho, 2013). En Colombia, para los territorios indígenas, los recursos pesqueros son un claro ejemplo de bienes comunes, definidos como los bienes que pertenecen a una colectividad, los cuales no son sujetos de apropiación individual, constituyen el modo de vida de la sociedad y representan el patrimonio socio-ecológico del territorio (Ostrom, 2000).

Los bienes comunes fueron inicialmente analizados por Garett Hardin (1968), en su teoría La tragedia de los Comunes, donde se mostraba como éstos eran usados de manera insostenible ante situaciones de escasez, o cuando no existían sistemas de control para su uso, quedando condenandos a la sobreexplotación, el agotamiento y la extinsión (Trujillo \& Escobar, 2015). En respuesta a los planteamientos de Hardin, Elinor Orstrom (2000) desarrolla una teoría complementaria, donde a partir de la recopilacion de experiencias en campo alrederor de todo el mundo, identificó las circunstancias que generan las diferentes formas de uso de los bienes comunes, y las razones que motivan a usarlo de maera colectiva, cooperativa o egoísta, de forma tal que se generen situaciones de sostenibilidad, resiliencia o agotamiento dentro de ellos. Ostrom señala que es posible generar acuerdos o contratos que involucren a todos los actores, generando estrategias de cooperación colectiva propia, con incentivos internos para su cumplimiento (Ostrom, 2000).

Este artículo presenta los resultados de un proyecto de investigación-acción-participativa, desarrollado por la Fundación Omacha, con el objetivo de crear estrategias para contrarrestar los problemas de agotamiento del recurso pesquero de los lagos de Tarapoto en el resguardo Ticuna, Cocama, Yagua, Ticoya y consolidar estrategias propias para fortalecer la capacidad de gobernanza interna del recurso pesquero dentro del territorio indígena.

El artículo se presenta en 7 secciones, que recogen el contexto local, la metodología empleada y los resultados obtenidos durante la creación y funcionamiento de los acuerdos. Asi, la primera seccion describe la problemática pesquera, su contexto local y la importancia de los acuerdos de pesca en la gestión de los bienes comunes; la segunda parte presenta la planeación y el proceso de formulación de los acuerdos al interior de la comunidad; la tercera describe la puesta en marcha de los acuerdos y la creación de un sistema de promoción, monitoreo y control local; la cuarta sección muestra los resultados del proceso tras seis años de funcionamiento; la sección cinco presenta las conclusiones y lecciones aprendidas durante el proceso, y finalmente la sexta parte presenta las recomendaciones para la gestión del recurso pesquero.

\section{La problemática pesquera y su contexto local}

El complejo de humedales y Lagos de Tarapoto, representa un territorio de importancia vital para el resguardo Ticoya y un ecosistema de alta fragilidad ambiental para la Amazonia colombiana. Está conformado por dos unidades ecológicas funcionales: la vársea, en los tramos afectados por las inundaciones 
periódicas del río Amazonas y el Igapó, correspondiente a los sectores influenciados por las aguas negras del río Loretoyacu. Adicionalemente, dentro del complejo se encuentran tres tipos de bosque: el de tierra firme que no presenta inundaciones; el bosque de vársea sujeto a inundaciones periódicas por aguas blancas, y el bosque pantanoso o lgapó que se inunda estacionalmente por aguas negras (TrujiIlo \& Duque, 2014). Dentro del bosque inundado se encuentran los pepeaderos, zonas del bosque con una alta producción de semillas, que son en su mayoría alimento alóctono para peces, por lo que el cuidado integral del ecosistema de bosques inundables es fundamental para garantizar la producción pesquera (Trujillo \& Trujillo , 2009). La Figura 1 presenta la ubicacion de los humedales y lagos de tarapoto y el área de influencia de los acuerdos de pesca y el programa de monitoreo.

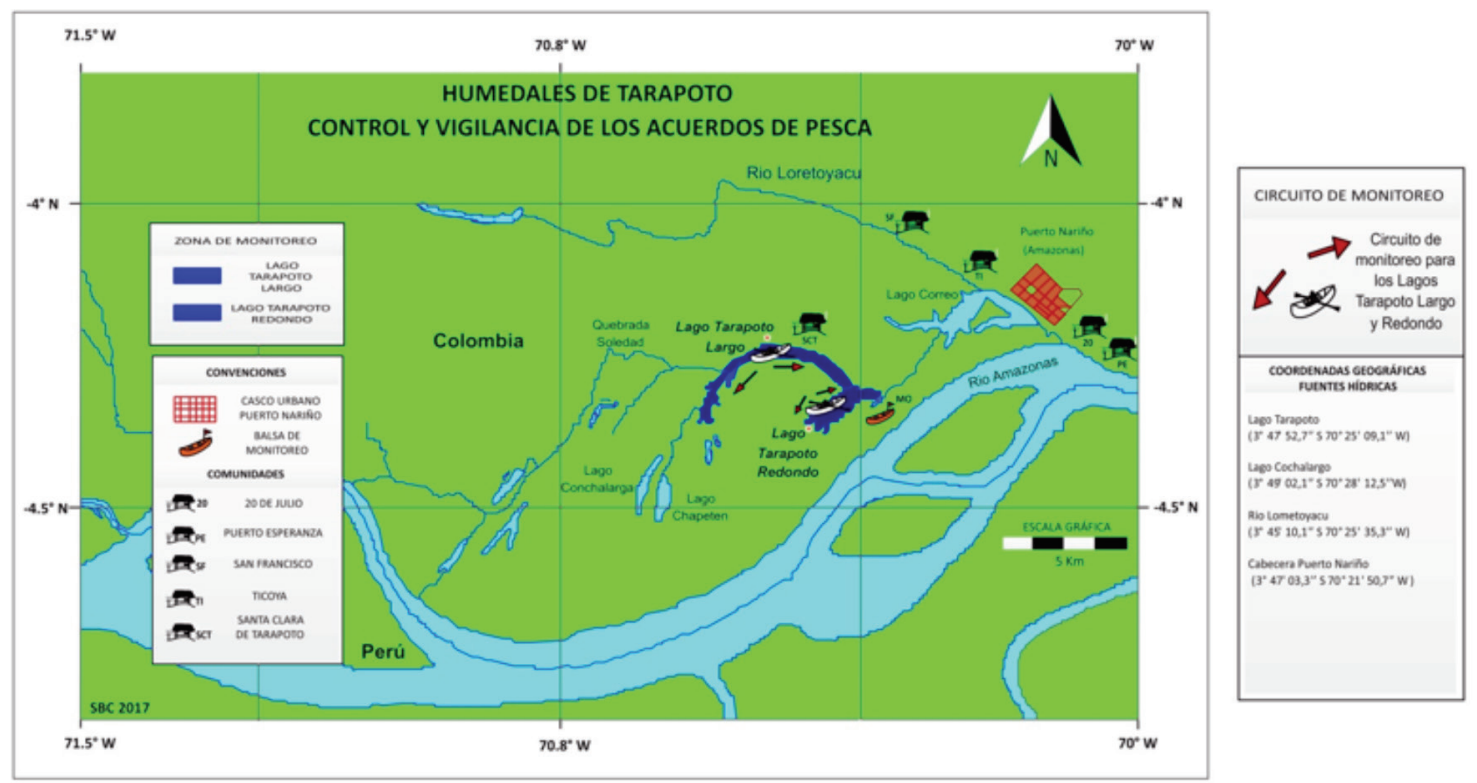

Figura 1. Lagos de Tarapoto y sistema de monitoreo y control de los acuerdos de pesca

Este ecosistema alberga 176 especies de peces, 244 de aves, 30 de reptiles, 56 de anfibios, y 76 de mamiferos y sustenta una población humana de más de siete mil personas que habitan en las comunidades aledañas al sistema (WWF- Fundación Omacha, 2017). Las actividades económicas del municipio son principalmente de tipo extractivista predominando la pesca, la extracción madera, el uso de productos del bosque y en menor medida el turismo. Por esta razón la disponibilidad de recursos naturales es de gran importancia, ya que estos garantizan una fuente de empleo e ingresos constante a las comunidades del municipio (Trujillo, 2014). Dentro de las actividades generadoras de ingresos, la población depende fuertemente de la actividad pesquera para garantizar su subsistencia. De acuerdo a estudios realizados por Trujillo \& Florez (2016), la pesca ocupa el segundo renglón dentro de la economía de los hogares indígenas, aportando el $24 \%$ de los ingresos, $10 \%$ por venta y comercialización de productos pesqueros y $14 \%$ por el autoconsumo directo del grupo familiar del pescador.

Aunque durante muchos años los habitantes de las comunidades usuarias del lago hicieron uso racional del ecosistema (Camacho, 2013; Durrance, 2003), factores recientes como el incremento poblacional 
y la introducción de nuevas tecnologías pesqueras como cuartos fríos y grandes mallas, han generado conflictos de uso del recurso, además de escasez y preocupación en la población indígena por el incremento en la presión pesquera y la reducción de la capacidad de resiliencia de los humedales y el lago de Tarapoto, como se identifica en los estudios realizados por Duque et.al (2009).

\section{Formulación de acuerdos de pesca}

La formulación de acuerdos de pesca inicia en el año 1992 con la expedición de la resolución No. 021 del INCORA del 13 de marzo de 1990, por medio de la cual se crea la propuesta para la reglamentación pesquera del municipio y resguardo de Puerto Nariño, formulado por el INPA, la cual nunca fue implementada por las autoridades del municipio, debido a la falta de acompañamiento de las instituciones administrativas de la región y por no haber abordado una aproximación participativa para su realización (Fundación Omacha; ICA, 2009).

Dado que en el trapecio amazónico la presencia de las organizaciones estatales competentes en la regulación de la normatividad pesquera es débil, en Puerto Nariño es fundamental que las comunidades ejerzan mecanismos propios de control territorial para abordar los problemas de gobernanza pesquera. De este modo, con la formulación de acuerdos de pesca se dio inicio a una etapa de consolidación de sistemas de manejos propios, diferentes a los practicados por el Estado colombiano, donde la población usuaria es quien asume la gobernanza de los recursos del territorio. Este pensamiento de gestión colectiva se materializa en el año 2005 con la formulación del plan de vida indígena del resguardo Ticoya, donde se establecieron lineamientos iniciales para el manejo de los recursos naturales del resguardo y se decretó a los lagos de Tarapoto como zona de manejo especial indígena
(ATICOYA, 2007). Posteriormente, en el año 2006 Corpoamazonia ordena la formulación del plan de manejo de humedales de Tarapoto, el cual se realiza de la mano con la Universidad Nacional de Colombia sede Amazonia, la Fundación Omacha y las autoridades del resguardo, a partir del cual se evidencia la necesidad de consolidar sistemas propios de gestión y control de los humedales del resguardo (Universidad Nacional de Colombia, Corpoamazonia, Fundación Omacha, 2007).

El inicio del proceso de creación de los acuerdos de pesca, se realizó a partir de actividades de investigación participativa con las autoridades locales, los pescadores y abuelos del resguardo. A partir de talleres comunitarios y trabajo colectivo se identificó la estrategia deseada para la gestión, manejo y control del recurso pesquero, de forma que esta fuera incluyente, concertada, factible y real para el contexto sociocultural y ambiental del resguardo (Duque et.al, 2009). De manera participativa se identificaron los pasos a desarrollar con la comunidad para consolidar la estrategia y su permanecia en el largo plazo (Trujillo \& Trujillo, 2009). Los pasos definidos fueron: i-Generar conciencia sobre los problemas que sufren los lagos de Tarapoto, ii- Invitar y motivar a la población para que participara activamente en la construcción de soluciones, iii-Informar y enseñar a la comunidad las formas como se pueden cuidar y manejar los lagos y los peces, iv-Organizar el pensamiento y las ideas, siguiendo el consejo de sabedores, abuelos y pescadores; v- Elaborar las propuestas de manejo y control que posteriormente se conviertieron en acuerdos formales de pesca, vi- Crear un sistema de monitoreo para fomentar el cumplimiento de los acuerdos y el monitoreo del lago (Trujillo \& Trujillo , 2009).

La Figura 2 presenta los pasos y elementos identificados para la elaboración de los acuerdos de pesca. 


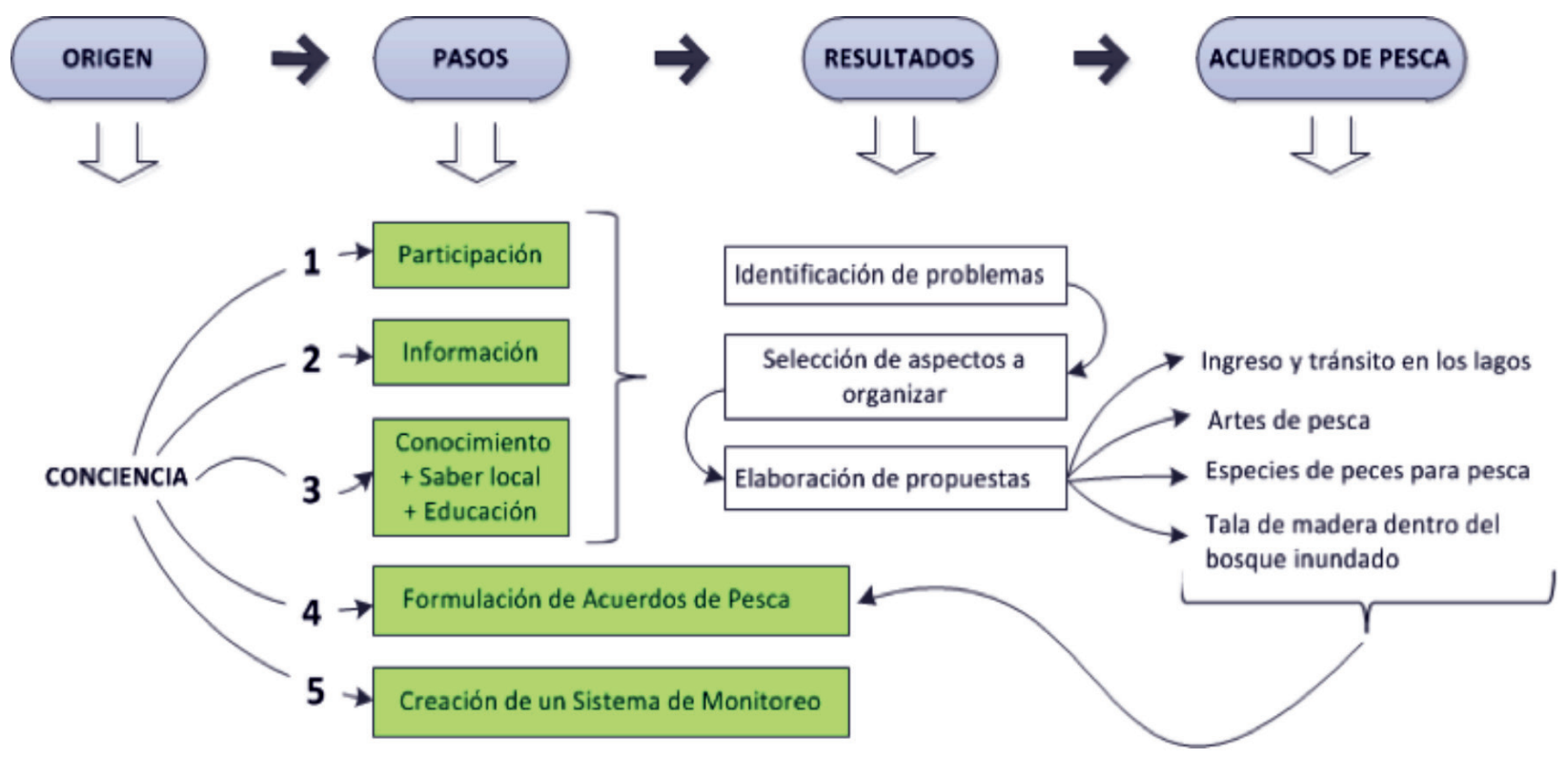

Figura 2. Pasos para la formulación de acuerdos de pesca de los lagos de Tarapoto

Como resultado de los talleres de construcción, se establecieron cuatro grupos de acuerdos o reglas, encaminadas a la solución de las principales problemáticas:

a. Manejo del recurso pesquero: establecen las especies y cantidades permitidas para la extracción, los periodos de veda y el control a la comercialización de alevinos y ornamentales.

b. Las artes de pesca: regulan el uso de mallas y elementos de pesca según el ciclo hidríco; prohiben el uso de elementos tóxicos y armas de fuego.

c. El ingreso al lago: establecen la velocidad de tránsito por los lagos y el ingreso de barcos pesqueros según su tipo; prohiben el ingreso de varcos pesqueros comerciales.

d. Manejo del lago y el bosque inundado: establecen medidas sobre el manejo especial de los pepeaderos y el bosque inundado; regulan la extracción forestal.

En el 2011, los acuerdos de pesca se presentan a la Asamblea Wone -maxima institucion legislativa del resguardo Ticoya-, para su validación como leyes propias derivadas de la sabiduria y el pensamiento indígenas, y son oficializados dentro del resguardo como normas internas de obligatorio cumplimiento para sus habitantes. Este reconocimiento por parte de la Asamblea Wone constituyó el inicio de la formalizacion juridica del proceso y la lejitimacion social del mismo, asi como la incorparacion del proceso al plan de vida indigena y la incorporacion de un sistema externo en la concepcion local del manejo del medio, lo que evidencia la transicion hacia sistemas hibridos de gestion territorial.

Posteriormente, en 2017 los lagos de Tarapoto son decretados como sitio RAMSAR consolidando un nuevo escenario para el manejo del territorio del resguardo Ticoya y sus recursos acuaticos. Adicionalmente en Junio de 2016, los acuerdos de pesca son reconocmidos por la Autoridad Nacional de Acuicultura y Pesca-AUNAP y obtienen resolución como normativa de obligatorio cumplimiento dentro del país, iniciando una nueva etapa para la gobernanza pesquera de la region (Resolución 1225 de 2017). La Figura 3 presenta la evolucion cronologica de la costrucción de los acuerdos de pesca como sistema de gestión pesquera. 


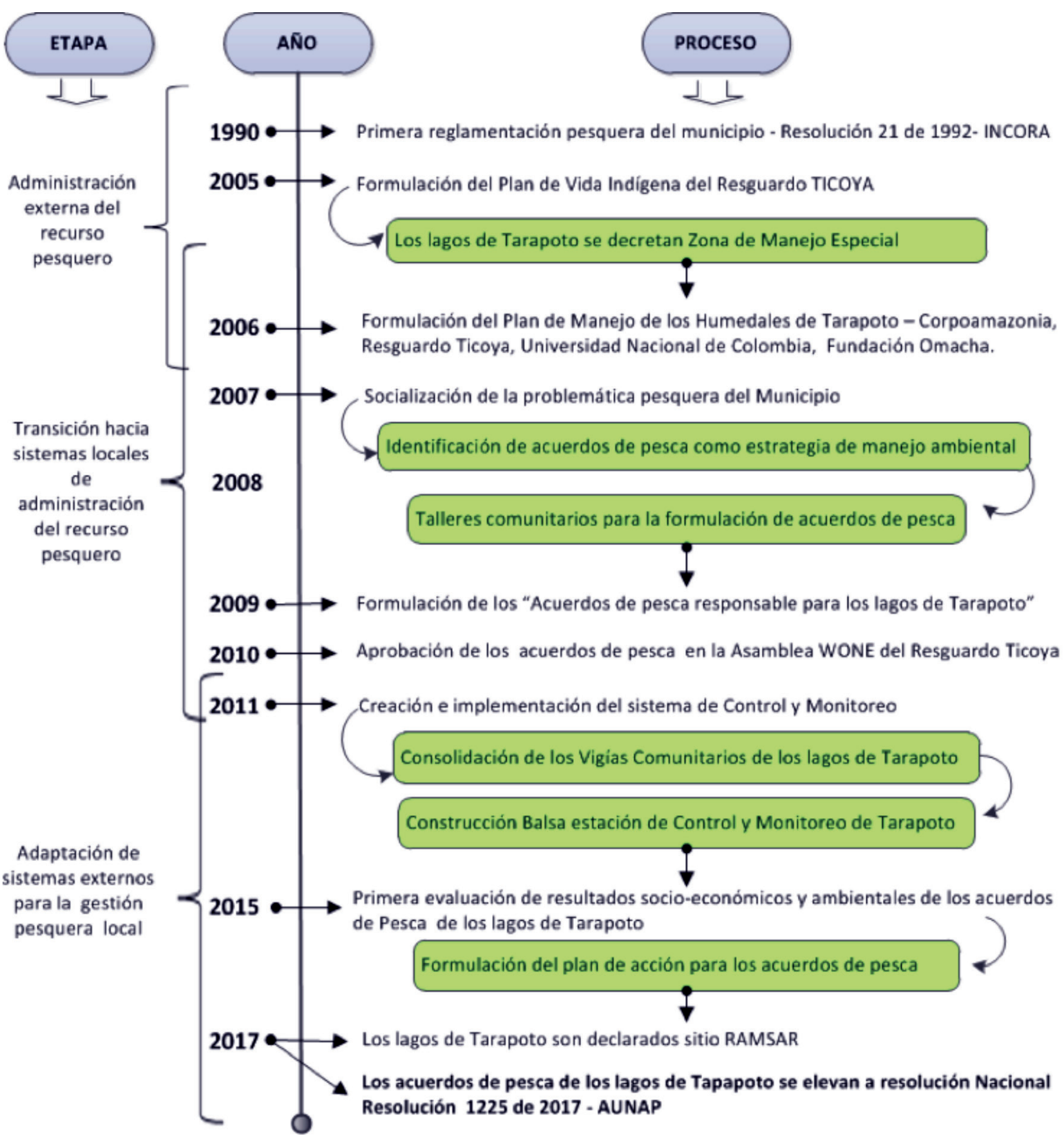

Figura 3. Origen y evolucion de los acuerdos de pesca de los lagos de Tarapoto.

\section{Implementación y control de los acuerdos} de pesca

Con el fin de implementar los acuerdos aprobados en la Asamblea Wone, se creó el programa de Vigías Comunitarios del Lago de Tarapoto, conformado por un grupo organizado de pescadores de las comunidades donde habitan los principales usuarios del recurso. La Tabla 1 muestra la ubicación de las comunidades usuarias del lago, la distancia de las mismas a la entrada del lago Tarapoto y el número de vigías por comunidad. 
Tabla 1. Comunidades usuarias y vigías del lago Tarapoto.

\begin{tabular}{l|l|c|c}
\hline \multicolumn{1}{c}{ Comunidad } & \multicolumn{1}{c}{ Ubicación de la comunidad } & Distancia al lago & No de vigías \\
\hline Puerto Esperanza & Ubicada sobre el río Amazonas & $9,30 \mathrm{~km}$ & 6 \\
\hline 20 de Julio & Ubicada sobre el río Amazonas & $8,41 \mathrm{~km}$ & 6 \\
\hline Puerto Nariño & Ubicado sobre el río Loretoyaco & $6,59 \mathrm{~km}$ & 3 \\
\hline Ticoya & Ubicada sobre el río Loretoyaco & $6,18 \mathrm{~km}$ & 3 \\
\hline Santa Clara de Tarapoto & Ubicada sobre lago Tarapoto Largo & $2,0 \mathrm{~km}$ & 4 \\
\hline San Francisco & Ubicada sobre el río Loretoyaco & $6,16 \mathrm{~km}$ & 4 \\
\hline
\end{tabular}

El grupo de vigías cumple la función de promotores ambientales e investigadores locales, realizando labores de monitoreo y promoción de los acuerdos de pesca con el resto de la comunidad que hace uso de los recursos del lago. Las actividades de monitoreo son realizadas mediante un sistema de turnos rotativos, que opera en una balsa flotante ubicada a la entrada de los lagos Tarapoto largo y Tarapoto redondo, durante las 24 horas al día, siete días a la semana. El monitoreo es complementado con recorridos diurnos y nocturnos por los lagos principales, con el fin de sensibilizar y educar a la población para que se cumplan los acuerdos de manera voluntaria y se promuevan las prácticas de pesca artesanales propias de la cultura indígena.

Respecto a las funciones de control, se realiza un registro de infracciones, siendo la más frecuente el uso de mallas no reglamentadas; hasta 2016 el cumplimiento de los acuerdos estaba sometido a la voluntad de los usuarios, dado que los vigías no tienen competencia para aplicar sanciones $u$ otras medidas correctivas, aspecto que durante los primeros años del programa constituyó un limitante para la eficacia del control y debilitó el proceso por la falta de autoridad local sobre los diversos usuarios del recurso; a la fecha los acuerdos son de obligatorio cumplimiento y deben ser respaldados por la autoridad nacional de pesca.

\section{Resultados de los acuerdos de pesca}

En 2015 se llevó a cabo la primera evaluación de resultados del programa. El seguimiento se realizó mediante una serie de encuestas a los pescadores vigías, monitoreo a la estación de control y visitas a los lagos; lo que permitió identificar las transformaciones en el manejo pesquero generadas a partir de la implementación de los acuerdos y del trabajo de los vigías comunitarios. Para la evaluación se recopiló información sobre los hábitos de pesca, las artes y aparejos utilizados y el producto por unidad de esfuerzo. Sobre los resultados de los acuerdos, se evalúo el cumplimiento de las metas iniciales teniendo en cuenta la percepción de los usuarios sobre los efectos de los acuerdos en el bienestar del recurso pesquero; también se analizó el funcionamiento administrativo del programa y las dificultades presentadas en el proceso. Las encuestas e inspecciones fueron aplicadas entre febrero y abril de 2015 -periodo de aguas en ascenso-, complementando la información con monitoreos semanales en la estación de vigilancia y control.

Sobre los hábitos y resultados de la pesca se identificó que cada pescador, extrae en promedio 4,46 sartas de pescado, destinado en un $68 \%$ al autoconsumo familiar, $3 \%$ a la redistribución comunitaria y $29 \%$ a la venta del producto en la comunidad. En cuanto a las artes utilizadas, se encontró que con la implementación de los acuerdos los usuarios cambiaron sus hábitos de pesca, viéndose reflejado principalmente en los métodos utilizados para extraer el pescado; la flecha, la vara y la maIla, elementos permitidos en los acuerdos, aparecen como los aparejos con mayor frecuencia de uso: flecha y vara $61 \%$, malla de ojo grande $31 \%$, 
otros $8 \%$. Adicionalmente, para el periodo analizado no se encontró ningún registro de elementos prohibidos, tales como las mallas menuderas que eran sin duda una de las principales causas de sobrepesca y reducción de la oferta pesquera. En cuanto a esto, el uso de las mallas aparece asociado a los niveles de inundación y a la capacidad de monitoreo de los vigías, especialmente en las zonas más apartadas y menos supervisadas de los lagos. Aunque los registros corresponden a mallas permitidas -agujero de malla mayor a 3 pulgadascontrolar el uso de éstas representa una de las mayores dificultades actuales, puesto que durante el ascenso de aguas los pescadores puede ingresar a los lagos por diferentes varaderos del bosque inundable, evadiendo el paso por la estación de control, e ingresando mallas no permitidas.

Respecto al cumplimiento de los acuerdos, se encontró que la mayoría de los pescadores tienen un mayor compromiso y disposición a cumplir las normas establecidas cuando se han desempeñado como vigías. Según registros, los pescadores vigías hacen mayor uso de elementos de pesca artesanales y en menor medida de mallas, que los pescadores no participantes del programa. La Figura 4 muestra los elementos de pesca empleados por los pescadores en el lago Tarapoto, teniendo en cuenta su participación en el programa de vigías.

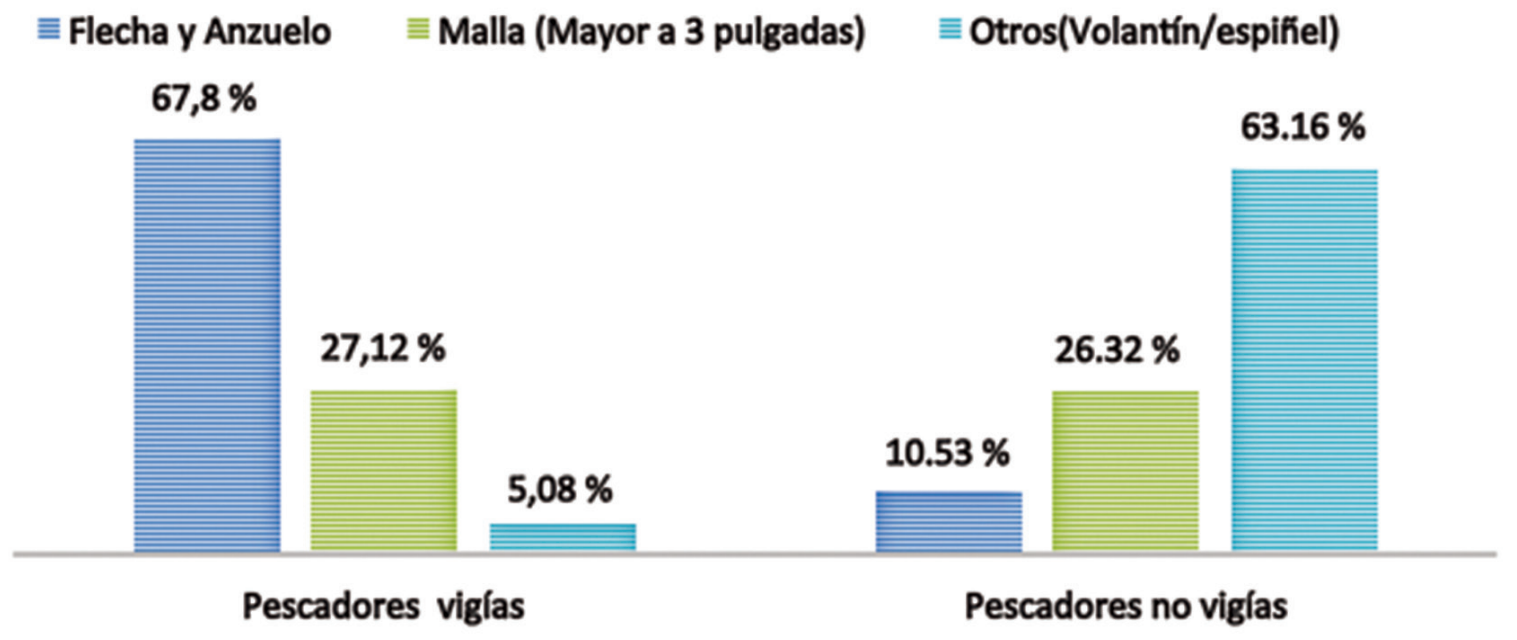

Figura 4. Elementos de pesca utilizados en los lagos de Tarapoto

Con relación al cumplimiento de los objetivos del programa, se encontró que los acuerdos han contribuido a la recuperación de la calidad ambiental del lago y los recursos pesqueros. De acuerdo a los resultados de las encuestas, tras cinco años de monitoreo y promoción de los acuerdos, la población reporta un incremento generalizado en las tallas de captura en especies como el Pirarucú Arapaima gigas, la Arawana plateada Osteoglossum bicirrhosum, la Gamitana Colossom spa y los Bagres Pseudoplatystoma sp (Figura 5), al igual que una mayor presencia de diversas especies de captura en general. Así mismo se reporta una diminución en el tiempo dedicado a las faenas de pesca, así como una mayor posibilidad de éxito en la pesca con artes tradicionales. La Figura 5 muestra la percepción de la comunidad sobre el incremento en las tallas de captura de cada especie. Según encuestas la Arawana plateada (Osteoglossum bicirrhosum) presenta la mayor recuperación del recurso con $42 \%$ de las encuestas reportando un incremento en su talla de captura. 


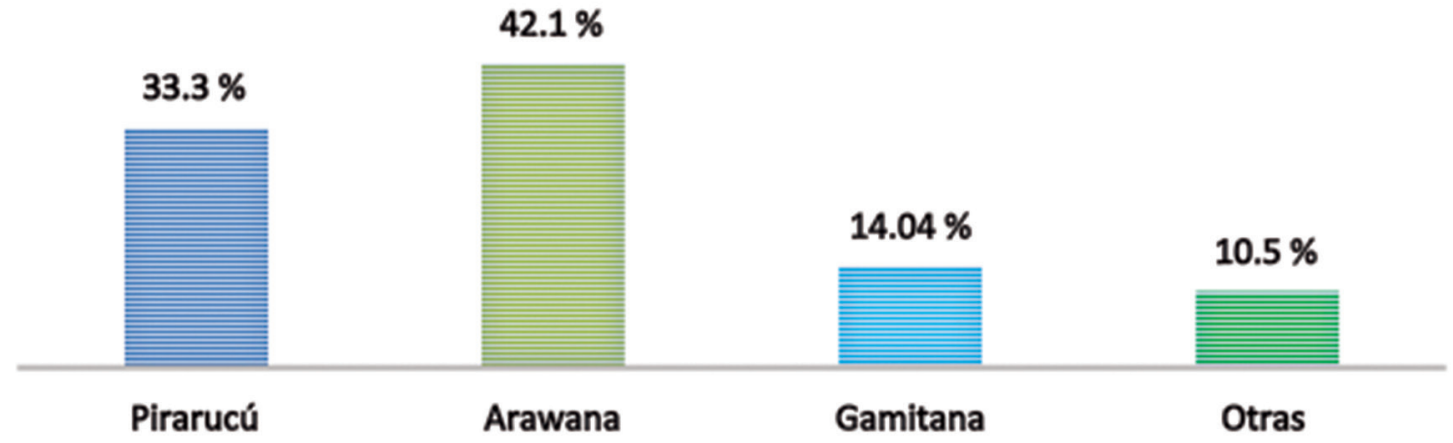

Figura 5. Percepción social sobre incrementos en las tallas de captura por especie.

Finalmente se encontró que, a nivel administrativo, la implementación de los acuerdos de pesca requiere de recursos permanentes para su financiación, que aseguren el monitoreo y las actividades de promoción y educación ambiental. Según encuestas, las autoridades del resguardo no cuentan con la capacidad administrativa necesaria para gestionar recursos financieros, ni para mantener activo el sistema de monitoreo, por lo que la presencia de recursos y actores externos se torna importante para garantizar la operatividad y continuidad del proceso de gestión propia del territorio, creando una figura de dependencia externa en la gobernanza local.

\section{Conclusiones y lecciones aprendidas}

Durante los últimos años, los acuerdos de pesca se han convertido en una herramienta real para el control y manejo del recurso pesquero en los lagos de Tarapoto, pues desde su implementación se ha evidenciado la recuperación de la oferta pesquera, se han restablecido las prácticas tradicionales de pesca y se ha retornado a la percepción de esta actividad como fuente de subsistencia familiar y provisión alimentaria. Hoy en día, los acuerdos son considerados por la población pescadora, como una herramienta que ha permitido la recuperación de los hábitos de pesca artesanales y los modos de vida tradicionales, generando conciencia en la comunidad acerca de la importancia de manejar y hacer un uso planeado de los recursos pesqueros y en general de los humedales de Tarapoto. De la información recolectada en el proceso de formulación, ejecución y seguimiento, se pudo evidenciar que los problemas relacionados al control y aplicación de sanciones son producto de dos factores: como primera medida, durante la planeación no se estableció con claridad quien debía asumir la responsabilidad de regular y supervisar el cumplimiento de los acuerdos, así como aplicar sanciones y medidas correctivas. Segundo, por ser un proceso en un resguardo indígena, las instituciones del Estado delegan la responsabilidad de gestión y control a la población y autoridades locales, de modo que el proceso carece de respaldo estatal.

Aunque la autoridad pesquera colombiana ha estado presente en las etapas del programa, especialmente en la legalización de los acuerdos, Resolución 1225 de 2017, su presencia y capacidad de reacción en la zona son limitadas por lo que los acuerdos de pesca carecen de un respaldo oficial real de hecho. Si bien este tipo de manejo es un proceso comunitario, la necesidad de apoyo estatal es una realidad, especialmente en una zona de frontera donde los límites internacionales no son respetados por los pescadores. Dada la ausencia de las instituciones del estado en la zona de estudio, y a la baja capacidad de gestión financiera de las comunidades locales, las ONG's han asumido el financiamiento operativo y las funciones logísticas de los acuerdos de pesca. De este modo, su presencia en el territorio se ha convertido en una fuente de financiación y gestión de los recursos pesqueros. De esta manera, el objetivo inicial de lograr una mejor autogestión de la pesca por parte de la comunidad y las autoridades locales del resguardo, no se cumple, pues las ONG's terminan asumiendo la 
responsabilidad de gestión de las autoridades tanto locales, como estatales.

Este proceso evidencia la relevancia de buscar sistemas alternativos de gestión territorial complementarios a la política de control de la autoridad pesquera nacional, y la importancia de fortalecer las capacidades de auto-gobernanza en las comunidades que buscan administrar sus territorios bajo parámetros propios. Durante la evolución del mismo, la sobrevivencia y evolución de los acuerdos de pesca evidenció la tendencia de la comunidad para adoptar sistemas híbridos de manejo del territorio, que, si bien fueron construidos de manera participativa, también presentan un alto contenido de pensamiento occidental basado en la planeación, el control, la supervisión y la sanción externa; elementos ajenos al sistema de manejo propio de la población local. La experiencia del proceso enseña que los acuerdos comunitarios pueden ser una estrategia para el uso sostenible del territorio, pero requieren de recursos financieros externos para garantizar los costos del monitoreo, las actividades de educación y la formación de capacidades de gestión; esto debido a que el cumplimiento de los acuerdos depende de supervisión constante, la cual es financiada con fondos externos generalmente gestionados por ONG's, lo que a través del tiempo va generando una dependencia de elementos externos al funcionamiento y el manejo tradicional del territorio.

\section{Recomendaciones para la gestión del recurso pesquero}

Se resalta la necesidad de un sistema de monitoreo con presencia local, pues la ausencia de una institución que se encargue de realizar funciones de control, es una dificultad que pone en riesgo la eficiencia de los acuerdos y del programa en general a pesar de su formalización como decreto nacional. Con este reconocimiento los acuerdos pasan a ser de obligatorio cumplimiento, tanto para la población local como para cualquier otro actor que quiera acceder al sistema de lagos, reforzando la capacidad de control y la eficiencia del sistema. Dado que los acuerdos no son solo reglas de pesca, sino que se entienden como todo un sistema de conservación del modo de vida tradicional indígena, el programa necesita de permanente acompañamiento y asesoría en diversos temas de educación ambiental y administración. Adicionalmente demanda estrategias de control y monitoreo directo de los lagos; por lo que no es posible concebirlo como un programa auto sostenible, pues requiere de recursos financiaros para su funcionamiento y no cuenta con ningún sistema para la generación de recursos propios.

Respecto al proceso de seguimiento y evaluación, aunque se encuentran resultados positivos, reflejados en la recuperación de especies, y mejora en las condiciones del ecosistema, se requiere la aplicación de seguimientos y análisis periódicos, que permitan identificar las fortalezas y dificultades del proceso y direccionarlo objetivamente. Finalmente, se requiere articular estas estrategias con las acciones, políticas y programas de manejo ambiental del municipio y el departamento, pues no hay una articulación con los planes de desarrollo municipal o departamental, no se cuenta con apoyo presupuestal de estas actores, y no hay integración entre el programa y otras líneas de gestión territorial en la región. Esta integración y articulación con el ente gubernamental local es clave para facilitar y mejorar el funcionamiento y control del programa y para el desarrollo futuro de los acuerdos de pesca.

\section{Agradecimientos}

Este artículo fue desarrollado dentro del marco del proyecto "Pensando, pescando y cuidando juntos; construcción de acuerdos colectivos para el manejo del recurso pesquero en territorios indígenas", desarrollado por la Fundación Omacha. Los autores agradecen a las diversas instituciones que apoyaron su realización; a los pescadores y sabedores que participaron en talleres y reuniones, a los vigías comunitarios de pesca, a las autoridades del resguardo Ticoya que han acompañado el proceso, y al equipo de la fundación Omacha por su apoyo y asesoría permanente. También se extiende un especial agradecimiento a las instituciones que financiaron las diversas etapas del proyecto: la Fundación Omacha, el INCODER, el ICA, la AUNAP, Corpoamazonia, la UNAL sede Amazonia, entre otros colaboradores. 


\section{Literatura citada}

1. Asociación de Autoridades Indígenas - ATICOYA (2007). Actualización del plan de vida de los Tikuna, Cocama y Yagua - Documento Síntesis - Plan de Vida 2007 -2017. 62 p. Recuperado de: http://bit.ly/2y0jHx9

2. Autoridad Nacional de Acuicultura y pesca - UNAP (2017). Resolución 1225 de 2017, Reglamentación de la actividad pesquera en los lagos de Tarapoto, departamento de Amazonas. Recuperado de http://bit. Iy/2yOQFNM

3. Barreto, C. \& Borda, C. (2008). Evaluación de los recursos pesqueros colombianos. Colombia: Instituto Colombiano Agropecuario ICA. 131p.

4. Caballero, G. \& Garza, M. (2010). La nueva economía institucional y la economía delos recursos naturales: comunes, instituciones, gobernanza y cambio institucional. Economía Agraria y Recursos Naturales, 10, 61-91.

5. Camacho, K. A. (2013). Tejiendo Sueños, atrapando peces: Hilando historias y conocimientos sobre el medio ambiente de la pesca en áreas de la Cuenca. Colombia. $255 \mathrm{p}$.

6. Castello, L. (2013). Perspectivas sobre el manejo comunitario de las pesquerías en la Amazonía. En Hacia el manejo de las pesquerías en la cuenca amazónica. Lima, Perú, Seminario Internacional Manejo Comunitario y Gobernanza Colaborativa de las Pesquerías en la Cuenca Amazónica, 2011. 94-100.

7. Duque, S. R., Trujillo Osorio, C., Huérfano, A., López Casas, S. \& Trujillo, F. (2009). Humedales amazónicos: experiencias de concertación para el manejo sostenible de territorios indígenas. En U. d. Zaragoza, Amazonia y agua. España: Servicio editorial de la Unesco.

8. Durrance, M. L. (2003). La naturaleza acuática en la vida social y cultural de los indígenas Ticuna del municipio de Puerto Nariño Amazonas. Bogotá, Colombia.

9. Fundación Omacha; ICA. (2009). Proyecto pensando, pescando y cuidando juntos: Propuesta para construir acuerdos colectivos para el manejo de recursos pesqueros en territorios indígenas. Leticia.

10. Gobernación del Amazonas. (2012). Plan de desarrollo del departamento del Amazonas (2012-2015). Recuperado de: $h$ ttp://bit.ly/1KUXc1V

11. Gutiérrez, F. D. (2010). Recursos hidrobiológicos y pesqueros continentales de Colombia. Colombia: Instituto de Investigación de los Recursos Biológicos Alexander von Humboldt. 118 p.

12. Lara, H. A. (2002). Reseña de "El gobierno de los bienes comunes. La evolución de las instituciones de la acción colectiva" de Elinor Ostrom. Región y Sociedad, 3.
13. Lasso, C., de Paula Gutiérrez, F., Morales, M. \& Agudelo, E. (2011). Pesquerías continentales de Colombia: cuencas del Magdalena-Cauca, Sinú, Canalete, Atrato, Orinoco, Amazonas y vertiente del Pacífico. Colombia: Instituto de Investigación de los Recursos Biológicos Alexander von Humboldt. 304 p.

14. Moreno, M. (2014). Contexto geográfico y social de los humedales de Tarapoto. En F. Trujillo, \& S. Duque, Los humedales de Tarapoto aportes al conocimiento sobre su biodiversidad y uso.

15. Olson, M. (1992). La lógica de la acción colectiva: bienes públicos y la teoría de grupos. México: Limusa. 75p.

16. Ostrom, E. (2000). El gobierno de los bienes comunes: la evolución de las instituciones de acción colectiva. México: Fondo de Cultura Económica de México. 65p.

17. Palma, L. (2014). Manejo integrado y sostenible de los recursos hídricos transfronterizos en la cuenca del Amazonas considerando la variabilidad y el cambio climático. Organización del Tratado de Cooperación Amazónica OTCA, Leticia. Recuperado de: http://bit. ly/2xmtOuf

18. Pantevis Girón, Y. (2013). Construyendo la historia ambiental de Leticia a través de la pesca. (Tesis Maestría). Universidad Nacional de Colombia. Leticia, Amazonas.

19. Piraquive, E. P. (2000). Estudio ictiológico de un caño de aguas negras de la amazonia colombiana, LeticiaAmazonas. (Tesis de Pregrado). Universidad Nacional de Colombia. Leticia, Amazonas.

20. ProVarzea. (2005). A pesca na região Amazonica. Projeto Manejo do recursos Naturales da Varzea. Brasil: Ibama-Brasil.

21. Trujillo, C. (2008). Selva y mercado: exploración cuantitativa de los ingresos en hogares indígenas. Tesis de Maestría. Leticia, Amazonas, Colombia.

22. Trujillo, C. (2014). Humedales de Tarapoto: Pesca, economía y seguridad alimentaria para el pueblo indígena de Puerto Nariño, Amazonas. En F. Trujillo, \& D. Santiago, Los humedales de Tarapoto: aportes al conocimiento sobre su biodiversidad y uso, 276-277.

23. Trujillo, C. \& Escobar, A. (2015). Restricciones conceptuales del ordenamiento territorial colombiano; usos del territorio y formas de propiedad. Entramado Vol 11, 2; 20-36.

24. Trujillo, C. \& Flórez, A. M. (2016). Contribución económica de la pesca artesanal a la economía de las comunidades ribereñas al Amazonas colombiano. Revista de Investigación Agraria y Ambiental RIAA, 7(1), 105-121.

25. Trujillo, C. \& Trujillo, F. (2009). acuerdos de pesca responsable para el buen uso de los lagos de tarapoto. Recuperado de: http://bit.ly/2xmsoor 
26. Trujillo, F. \& Duque, S. (2014). Los humedales de Tarapoto: aportes al conocimiento sobre su biodiversidad y uso. Recuperado de: $h$ ttp://bit.ly/2xInUOG

27. Universidad Nacional de Colombia Sede Amazonia. (2011). Desarrollo propio, co-manejo y sostenibilidad en los lagos de Yahuarcaca. Informe final, USAID del pueblo de los Estados Unidos de América, Leticia.

28. Universidad Nacional de Colombia, Corpoamazonia, Fundación Omacha. (2007). Formulación del Plan de Manejo de Humedales. Leticia.
29. WWF- Fundación Omacha. (2017). Estudio Técnico para la designación del nuevo sitio Ramsar complejo de humedales de Tarapoto. Recuperado de: http://bit. ly/2xztjTg

30. Zuluaga, P. A. \& Franco, M. (2014). Comportamiento de Pescadores frente a distintos arreglos institucionales en la Estrella Fluvial del Inírida. USAID del Pueblo de los Estados Unidos de América. Recuperado de: $h t t p: / /$ bit.ly/2xIIKP4
Conflicto de Intereses Los autores declaran no tener ningún conflicto de intereses

Recibido: Enero 26 de 2017 Aceptado: Marzo 13 de 2017 